\title{
Modélisation du devenir à court terme des boues de dragage rejetées par clapage
}

\author{
Ricardo Silva Jacinto ${ }^{1}$ et David Burel ${ }^{2}$
}

${ }^{I}$ Chercheur, Ifremer - Centre de Brest, DEL-EC-TP, B.P. 70, 29280

Plouzané.02.98.22.46.61. Ricardo.Silva.Jacinto@ifremer.fr.

${ }^{2}$ Ingénieur de l'Ecole Centrale de Nantes. 8, rue fontaine venise, 50000 Saint-Lô. 02.33.05.61.02. david burel@hotmail.com.

Résumé

Les boues de dragage présentent par fois une contamination en métaux lourds et contaminants organiques. La décision fréquente de rejeter ces boues en mer doit être basée sur une estimation des impacts de tels rejets dans la zone d'immersion (champ proche) et dans les zones avoisinantes (échelles locale à régionale). Cette étude présente une approche de modélisation du devenir à court terme des sédiments issus des dragages rejetés par clapage, à laquelle pourraient être couplés des modèles de la cinétique et de la dissolution propre aux contaminants associés.

Abstract

In France, contaminated dredge materials are frequently released from barges in open sea, near sensitive ecosystems. In order to assess the potential impact of such disposals, a short-term modelling approach for dredged sediments released from barges is proposed. This modelling approach is able to integrate contaminant kinetics and dilution models to provide an initial condition to regional and deterministic estimations of the pathways of both sediments and contaminants.

\section{Introduction}

Environ 50 millions de mètres cubes de sédiments sont dragués en moyenne, en France, et rejetés dans le milieu marin. Les volumes les plus importants de matériaux déplacés le sont par les grands ports qui rejettent, entre autres, plus de 15 millions de mètres cubes de vases par clapage (L'Yavanc et al., 1999).

Les boues de dragage peuvent contenir des métaux lourds, des contaminants organiques, une teneur en matière organique élevée, contiennent une quantité appréciable de sulfures et sont souvent anoxiques (Alzieu et al., 1999). Ces conditions favorisent l'immobilisation effective de nombreux contaminants, tant que le matériau de dragage n'est pas mis en suspension dans le milieu marin, par nature riche en oxygène. Ces sédiments présentent ainsi un potentiel important de relargage des contaminants quel que soit le mode de rejet ou dépôt choisi. Lors d'un rejet par clapage, les contaminants associés aux boues de dragage et, en moindre part, aux eaux interstitielles, sont ainsi susceptibles de se désabsorber des 


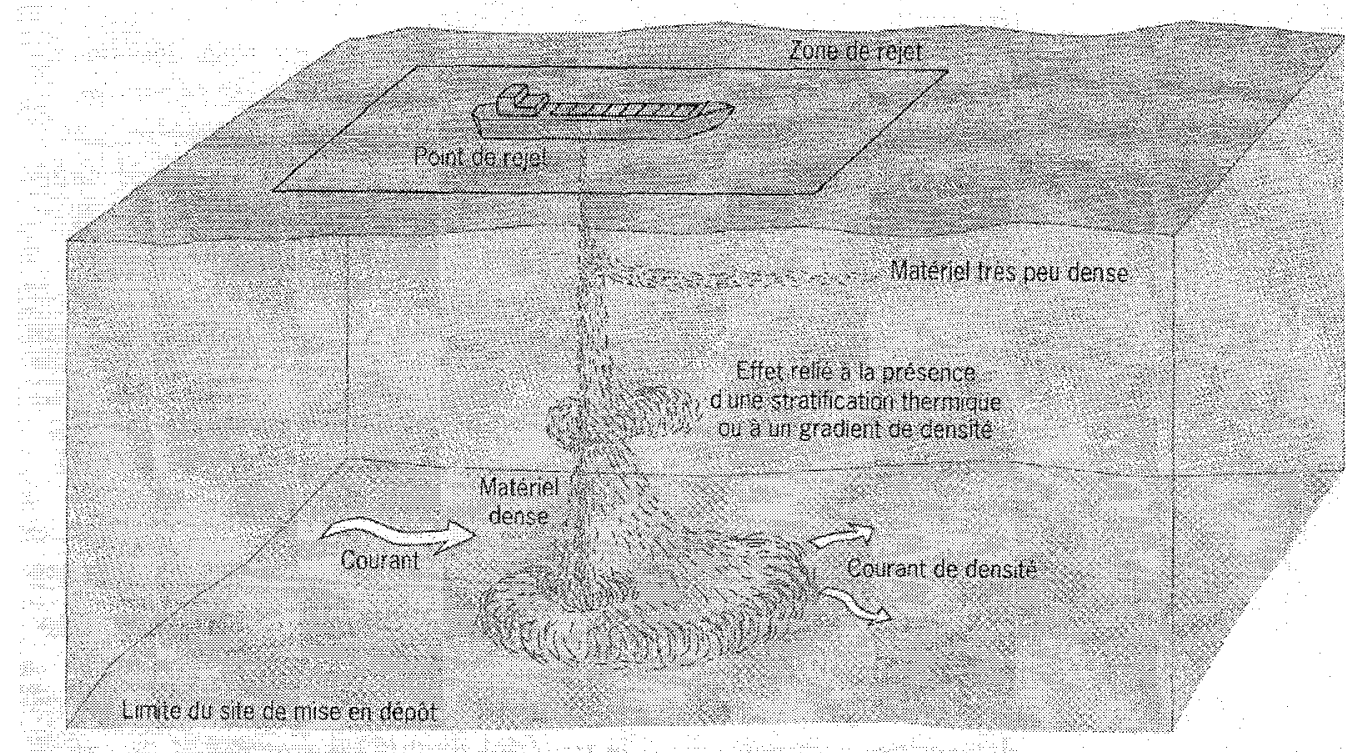

Figure 1 - Comportement des sédiments au cours d'un rejet de dragage par clapage (d'après Truitt, 1988).

sédiments rejetés dans les premières heures après un rejet et de se dissocier des sédiments de dragage. Il est ainsi important de pouvoir modéliser conjointement le devenir, à court terme et dans le champ proche, des boues de dragage et des contaminants associés.

Lors d'un rejet de dragage par clapage, le mouvement complexe, qui tend à projeter, plus ou moins rapidement, le matériau vers le fond, est habituellement décrit en deux phases : convection ou descente en masse, et effondrement dynamique (ou étalement) sur le fond (Silva Jacinto et al., 1999). Une fois le rejet étalé sur le fond, dans un état d'énergie minimum, son comportement devient indépendant de la technique de rejet. A partir de ce moment, l'évolution du matériau se réalise à long terme et peut être décrite par des modèles classiques d'advection-dispersion et transport sédimentaire. L'approche de modélisation présentée dans ce travail concerne les deux premières phases : la convection ou descente en masse et l'effondrement dynamique sur le fond (Figure 1).

\section{Modèle de convection}

Le modèle de convection simule la descente rapide sous l'effet de la gravité des matériaux clapés à partir d'une barge. Il est inspiré de la modélisation de la phase de chute convective utilisée par l'United States Army Corps of Engineers (USACE) dans son modèle de champ proche (STFATE). Les développements théoriques ont été initialement proposés par Koh et Chang (1973). Ces premiers travaux sont développés plus tard par Brandsma et Divoky (1976) et retraités de manière plus sommaire dans des textes des membres de I'USACE : McAnally et Adamec (1987), Johnson et al. (1995). Une version adaptée aux eaux profondes a été présentée par Abdelrhman et Dettmann (1993). Les modifications apportées dans le modèle présenté correspondent au 
remplacement d'une équation de flottabilité par un bilan de masse sédimentaire et par un critère différent de détachement des particules du nuage principal.

\subsection{Equations}

Le modèle présenté résout des équations intégrales, i.e. pour l'ensemble du nuage sédimentaire. Ces équations dynamiques conservent les quantités de base : la quantité de mouvement, la masse totale du rejet et la masse des sédiments. Les processus incorporés dans les équations tiennent compte, entre autres, de la dilution du nuage par entraînement durant la chute. Il permet ainsi de reproduire le mouvement de convection pendant lequel le nuage de matériau, de forme supposée a priori hémisphérique, descend dans la colonne d'eau sous l'effet de la gravité et de ses caractéristiques propres (densité, volume, forme...). Durant le mouvement de convection, il est admis qu'une partie du matériel se détache du nuage principal et demeure dans la colonne d'eau où les particules acquièrent un comportement individualisé dicté par leur vitesse de chute et l'hydrodynamisme environnant. Les mécanismes de détachement étant mal connus, dans la modélisation proposée, le volume susceptible de se détacher du nuage principal correspond à celui d'une couche extérieure où la concentration solide devient, par entraînement, inférieure à une concentration critique. Cette concentration critique correspond à la concentration au-delà de laquelle la chute des particules est entravée : les particules se gênent alors les unes les autres pendant la chute. Cette concentration critique d'entravement est ainsi prise comme critère de détachement car elle correspond en quelque sorte à une limite de concentration pour les interactions inter-particulaires. Sa valeur dépendra du matériau et de son organisation granulométrique ; les valeurs citées dans la littérature avoisinent les $10 \mathrm{~kg} \cdot \mathrm{m}^{-3}$ (Teeter, 1984). Le matériel détaché du nuage est artificiellement distribué sur l'incrément de profondeur correspondant au pas de temps de calcul, ce qui est justifié par les circulations derrière un nuage approximativement hémisphérique données par des modèles de convection de haute résolution à physique complète (Gotoh et al. 2000 ; Burel et Garapon, 2002), 3D et pression non hydrostatique.

Les équations du modèle, résolues par une décomposition explicite des dérivées temporelles en différences finies, s'écrivent dans leur forme différentielle :

$$
\begin{aligned}
& \frac{\mathrm{dm}}{\mathrm{dt}}=E \rho_{\mathrm{a}}-\sum_{\mathrm{i}} \mathrm{S}_{\mathrm{i}} \rho_{\mathrm{i}}-\mathrm{v}_{\mathrm{d}} \rho_{\mathrm{c}} \\
& \frac{\mathrm{d} \overrightarrow{\mathrm{M}}}{\mathrm{dt}}=\mathrm{F}_{\mathrm{c}}-\mathrm{F}_{\mathrm{D}}+E \rho_{\mathrm{a}} \overrightarrow{\mathrm{U}}_{\mathrm{a}}-\sum_{\mathrm{i}} \mathrm{S}_{\mathrm{i}} \rho_{\mathrm{i}} \overrightarrow{\mathrm{U}}_{\mathrm{a}}-\mathrm{v}_{\mathrm{d}} \rho_{\mathrm{c}} \overrightarrow{\mathrm{U}}_{\mathrm{c}} \\
& \frac{\mathrm{dV} \mathrm{c}}{\mathrm{dt}}=\mathrm{E}-\sum_{\mathrm{i}} \mathrm{S}_{\mathrm{i}}-\mathrm{v}_{\mathrm{d}}
\end{aligned}
$$

Dans ces équations, $\mathrm{m}, \mathrm{M}$ et $\mathrm{V}_{\mathrm{c}}$ représentent les trois grandeurs conservées : respectivement la masse, la quantité de mouvement et le volume. Les indices c, a, i, D et d se réfèrent respectivement au nuage, au milieu environnant, à la classe de particules, à la trainée hydrodynamique et au détachement des 
particules. Dans les paramètres restants, $v$ représente une variation de volume, $\mathrm{E}$ un taux d'entraînement, $S$ un taux de départ des matériaux du nuage principal par détachement, $\rho$ une densité, $F$ les forces agissant sur le nuage, $U$ la vitesse et $t$ le temps. Les équations (1), (2) et (3) permettent ainsi de calculer les inconnues du problème : la vitesse du nuage, sa densité et sa masse.

\subsection{Critère de détachement}

En raison de l'incorporation d'eau par entraînement, le rayon du nuage tend naturellement à augmenter et sa concentration moyenne en sédiments à diminuer. Lorsque, aux limites extérieures du nuage, la concentration devient inférieure à la concentration critique d'interaction particulaire, ces particules sont facilement enlevées et détachées du nuage par entraînement. Ce détachement correspond à la matière comprise entre un rayon interne au nuage $\left(\mathrm{R}_{\mathrm{d}}\right)$, au-delà duquel la concentration est inférieure à la concentration critique $\left(\mathrm{C}_{\mathrm{d}}\right)$, et le rayon extérieur du nuage $\left(R_{c}\right)$. A l'instar d'Abdelrhman et Dettmann (1993), pour la détermination du rayon de détachement, la distribution radiale de la concentration en sédiments du nuage est supposée Gaussienne :

$$
\mathrm{C}(\mathrm{r})=\mathrm{C}_{0} \cdot \mathrm{e}^{-\frac{\mathrm{r}^{2}}{\sigma_{0}^{2}}}
$$

Dans cette expression, $\mathrm{C}_{0}$ est la concentration initiale des matériaux, supposée conservée au cœur du nuage, $r$ la distance au centre du nuage, et $\sigma_{0}$ une échelle virtuelle de distribution telle que la quantité de sédiments dans le nuage avant détachement est conservée :

$$
\int_{0}^{\infty} 2 \pi r^{2} \cdot C(r) \cdot d r=\bar{C} \cdot V_{c}
$$

où $2 \pi \mathrm{r}^{2}$ correspond à l'aire de la surface de l'hémisphère d'iso-concentration de rayon $r$ et $\bar{C}$ est la concentration moyenne du nuage, connue à chaque instant. Le rayon de détachement est ainsi donnée par le rayon tel que $C\left(R_{d}\right)=C_{d}$. La masse de sédiments détachée du nuage à chaque pas de temps peut ainsi s'écrire :

$$
\mathrm{m}_{\mathrm{d}}=\frac{2 \pi}{3} \cdot \frac{\mathrm{m}_{\mathrm{c}}}{\mathrm{V}_{\mathrm{c}}} \cdot\left(\mathrm{R}_{\mathrm{c}}^{3}-\mathrm{R}_{\mathrm{d}}^{3}\right), \quad \mathrm{R}_{\mathrm{d}}<\mathrm{R}_{\mathrm{c}}
$$

\subsection{Résultats}

Le modèle a été validé par comparaison avec des mesures expérimentales présentées par Boutin (2000) et réalisées dans le canal 5 du Laboratoire National d'Hydraulique et Environnement (LNHE). Ce canal est large de 1,5 m, long de 80 $\mathrm{m}$ et sa hauteur est de $1 \mathrm{~m}$. Le bac de rejet, simulant une barge, a un volume de $0.06 \mathrm{~m}^{3}$ et est placé au-dessus de la surface libre. Les données ont été interpolées à partir de photographies prises durant les expériences (Boutin, 2000). L'exemple présenté sur la figure 2 concerne le test 11 dont le rejet présentait une densité de $1280 \mathrm{~kg} \cdot \mathrm{m}^{-3}$. 

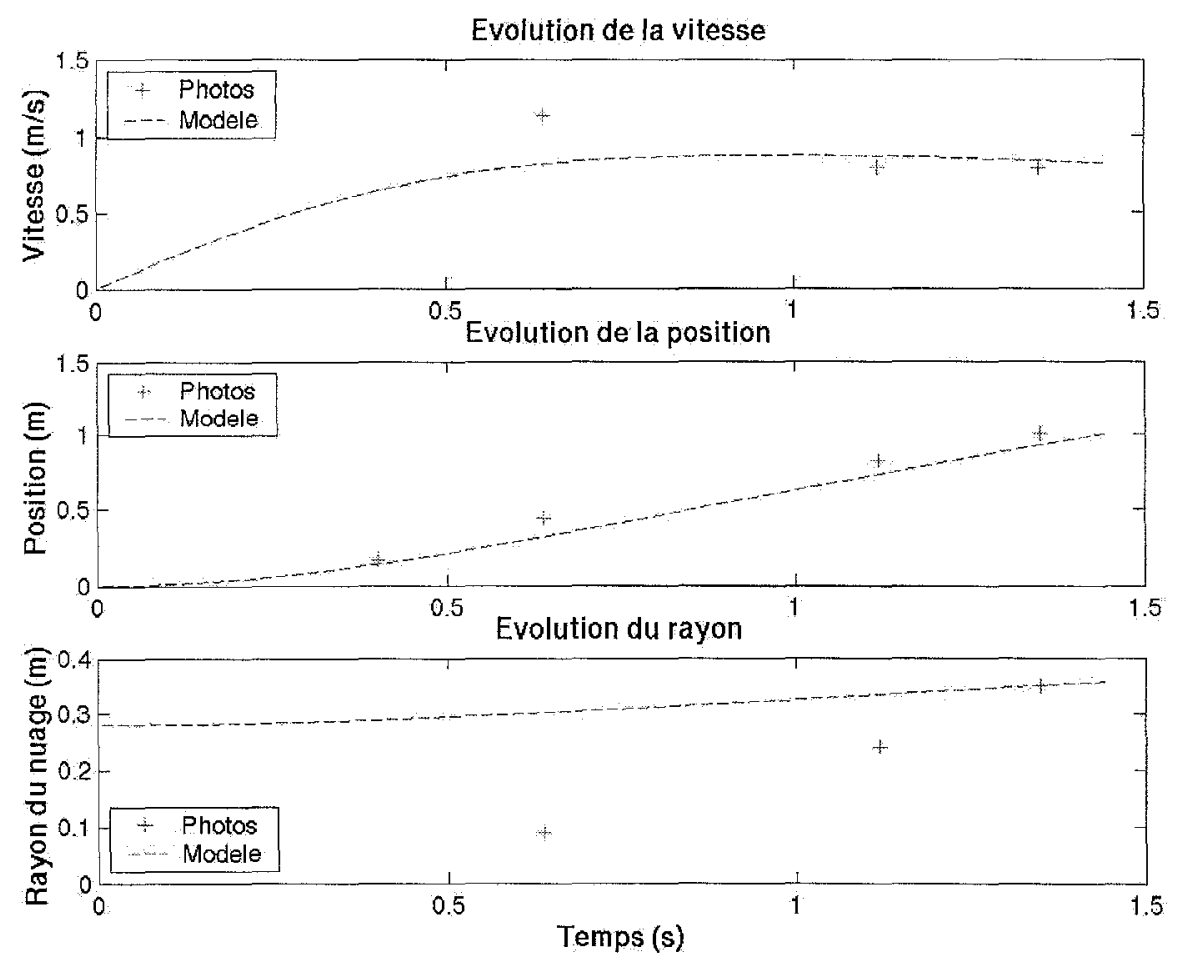

Figure 2 - Evolution temporelle des paramètres lors du test 11. Données : Boutin (2000).

Cet exemple montre que le modèle est capable de reproduire les paramètres cruciaux pour la dispersion d'un rejet: chute du nuage, vitesse d'impact près du fond, concentration ou dimension finale du nuage. Toutefois, les dimensions réduites des expériences de Boutin (2000) ne permettent pas que à tous les processus ayant lieu lors d'un rejet in situ s'établissent; notamment, la perte de matériaux par entraînement ne peut pas être validé par comparaison avec ce jeu de données. La simulation avec les paramètres calibrés grâce aux mesures de Boutin (2000) de rejets aux échelles proches des rejets usuels $\left(500 \mathrm{~m}^{3}, 15 \mathrm{~m}\right.$ de profondeur) donne cependant une perte de matériaux dans la gamme des 7 à $14 \%$ indiquée dans la littérature (Truitt, 1986).

\section{Modèle d'étalement sur le fond}

La phase d'effondrement dynamique correspond à l'interruption du mouvement de convection par un violent étalement horizontal sur le fond. Lors de l'effondrement, il y a formation d'un courant de densité qui s'étale radialement autour du point d'impact. Ce courant de densité, ou turbidité, est simulé à l'aide d'un modèle intégré sur la verticale qui résout par une technique de volumes finis les équations de conservation de la quantité de mouvement, de la masse totale et de la masse sédimentaire (Bradford et al., 1997). Ce modèle, où nous avons intégré l'effet non linéaire d'un courant ambiant, tient compte des divers processus en jeu : présence ou pas d'un courant ambiant, l'entraînement d'eau à la surface du courant de densité, la chute des particules, la turbulence, l'érosion et le dépôt des sédiments et l'évolution bathymétrique liée aux échanges avec le fond. Partant d'une condition initiale donnée par le modèle de convection, i.e. quantité 


\section{VII ìmes Journées Nationales Génie Civil - Génie Côtier, Anglet, France, 15-17 Mai 2002}

de mouvement, masse et volume, ce deuxième modèle permet d'obtenir la répartition horizontale des sédiments déposés et en suspension dans le courant de densité ainsi que la modification bathymétrique quand le courant de densité cesse.

\subsection{Equations}

Le détail des équations du modèle, ainsi que le schéma de résolution, est présenté et discuté dans Bradford et al. (1997). Les équations à la base du modèle sont les équations de conservation du sédiment, du fluide et de la quantité de mouvement en moyenne verticale. Ces équations des courants de turbidité forment un système couplé d'équations différentielles, non-linéaires qui sont mathématiquement similaires aux équations d'Euler compressibles. Ces équations sont valides pour des courants de turbidité 2D (moyennés sur la verticale), qui se déplacent dans un fluide de masse volumique constante qui serait au repos. Les améliorations apportées permettent de considérer le cas d'un fluide environnant en écoulement stationnaire et uniforme. Il s'agit d'une représentation mono-couche ; le courant se déplace au fond sous une couche très importante de fluide au repos ou pourvu d'un mouvement uniforme et stationnaire. Le système peut s'écrire sous une forme intégrale, proposée par Parker et al. (1986) :

$$
\frac{\partial \mathbf{U}}{\partial \mathrm{t}}+\frac{\partial \mathbf{F}}{\partial \mathrm{x}}+\frac{\partial \mathbf{G}}{\partial \mathrm{y}}=\mathbf{Q}
$$

Dans cette équation, $\mathbf{U}=(\mathrm{h}, \mathrm{hU}, \mathrm{hV}, \mathrm{hC})^{\mathrm{t}}$ représente le vecteur des variables conservatrices, i.e. dans l'ordre, l'épaisseur du courant de turbidité, les flux de quantité de mouvement dans les deux dimensions horizontales de l'espace et le flux de particules. Les vecteurs $\mathbf{F}$ et $\mathbf{G}$ contiennent les termes de transport advectif liés au courant de turbidité alors que le terme $\mathbf{Q}$ représente les sources favorisant ou entravant le courant de turbidité : l'entraînement, le facteur de pente, le frottement, l'érosion ou dépôt des sédiments. Les termes du transport advectif de la concentration en sédiments par le courant ambiant est, lui aussi, introduit dans les termes sources.

\subsection{Discussion et résultats}

Le modèle de courant de turbidité a été validé par reproduction des expériences de validation présentées par Bradford et al. (1997) et Bradford et Katapodes (1998). Il n'a pas été validé par rapport à des mesures de l'étalement sur le fond d'un rejet de dragage.

Comme exemple de résultat de ce type d'application, nous présentons deux simulations respectivement avec ou sans présence d'un courant ambiant et avec un fond horizontal. L'effet de pente du fond n'est pas présenté mais a été analysé avec le modèle.

Les résultats présentés sur les figures 3 à 5 concernent un rejet de $500 \mathrm{~m}^{3}$ s'étalant sur un fond plat en présence ou pas d'un courant ambiant de $10 \mathrm{~cm} \cdot \mathrm{s}^{-1}$ orienté de gauche vers la droite. Les résultats sont ceux obtenus après 2 min de simulation. On observe que la symétrie lors d'un rejet dans un milieu au repos est très modifiée en présence d'un courant ambiant. Dans un milieu au repos l'épaisseur du courant de turbidité est maximal au niveau du front. En présence de 


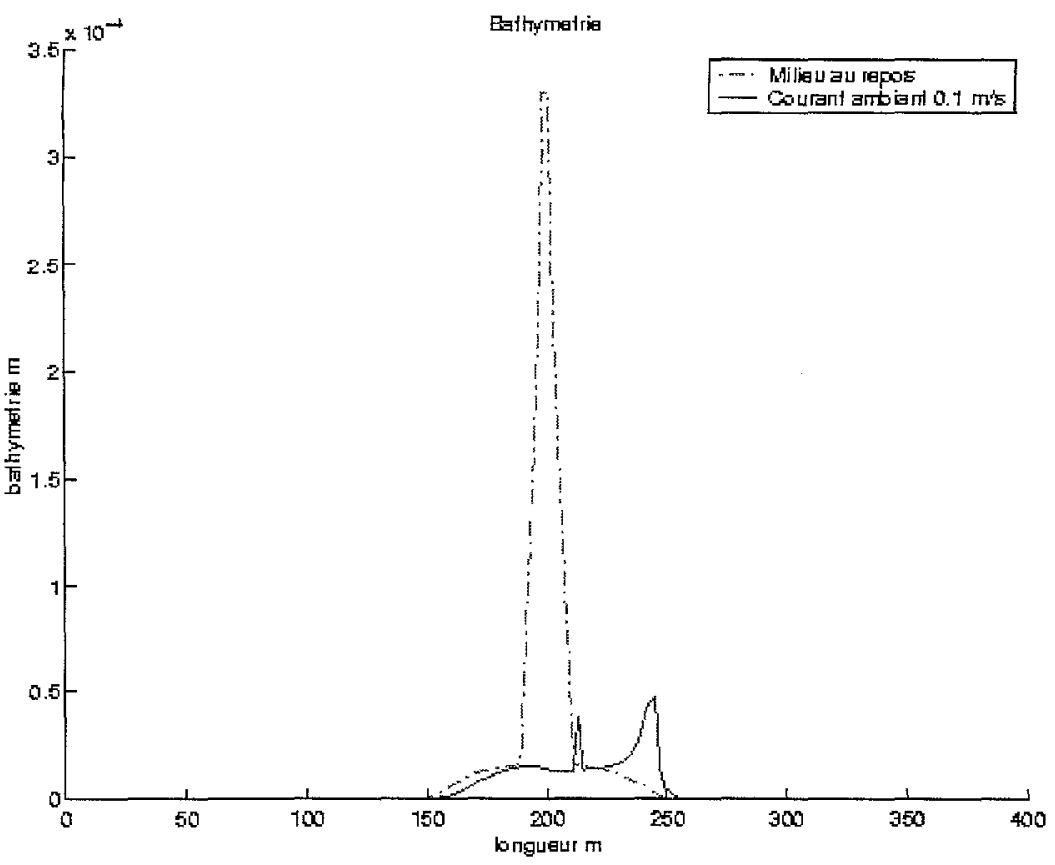

Figure 3 - Evolution bathymétrique après 2 min de simulation.

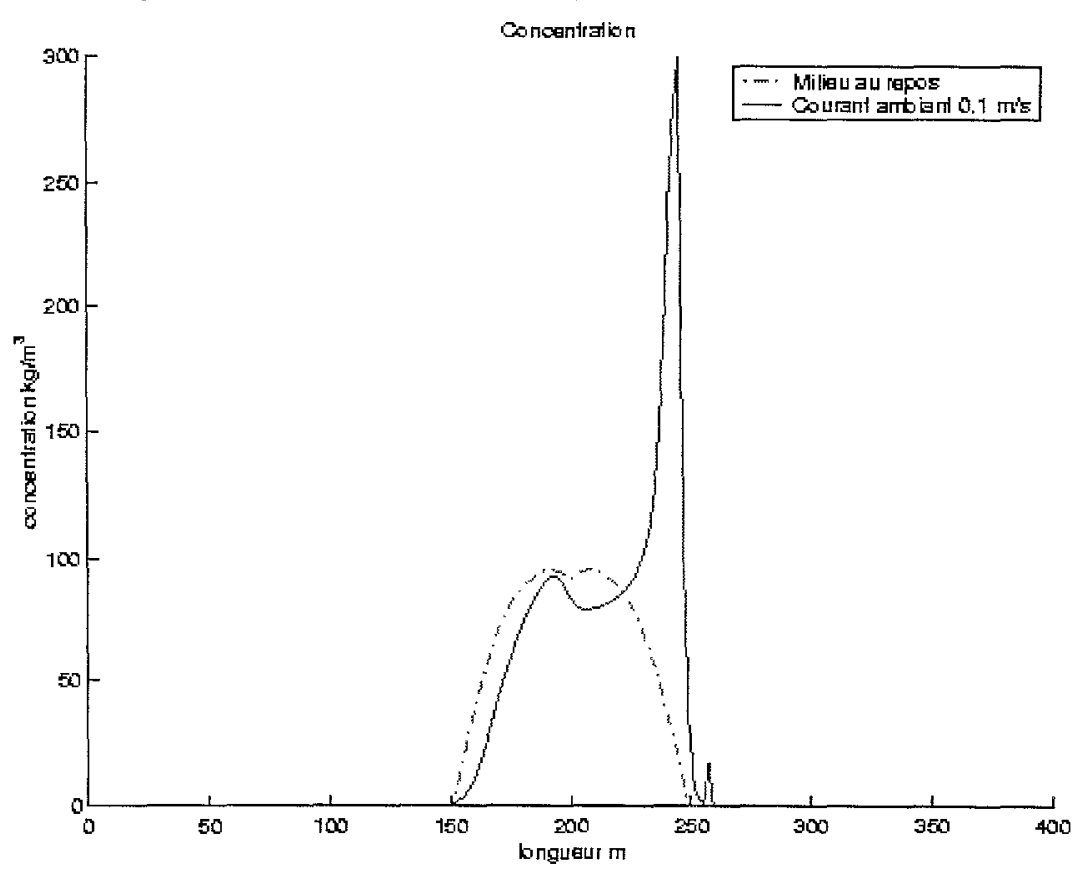

Figure 4 - Evolution de la concentration après 2 min de simulation.

courant cela reste vrai mais le front se propageant dans le sens du courant ambiant est inférieur au front s'opposant au courant ambiant : un phénomène de réfraction ou levée par le courant ambiant est observé.

Concernant la concentration au sein du courant de turbidité, nous pouvons observer une asymétrie très importante en présence d'un courant ambiant. Le front se propageant dans le sens du courant ambiant est beaucoup plus chargé en particules : nous pouvons donc nous attendre à une advection très importante de la 


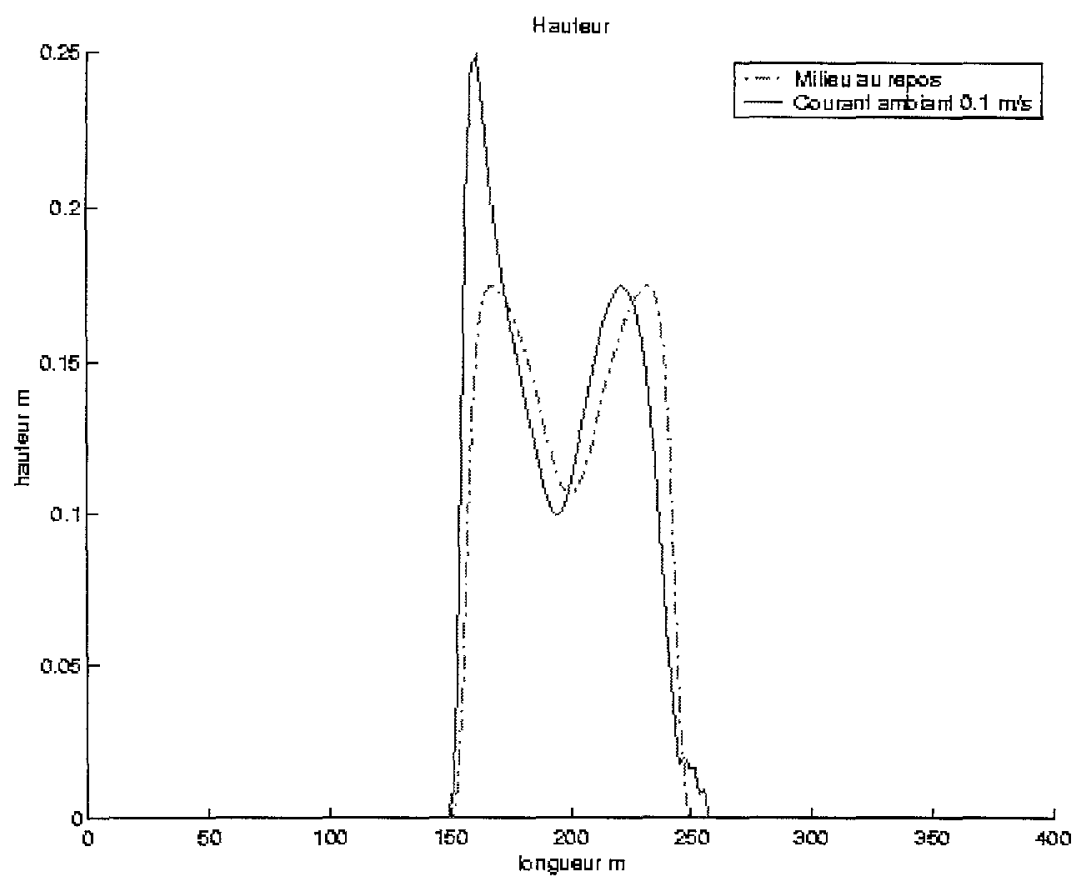

Figure 5 - Evolution de la hauteur du courant de turbidité.

masse turbide. Les dépôts observés après 2 min de simulation montrent en effet une tendance à un dépôt à l'extrémité aval du courant, alors qu'en absence de courant ambiant, le dépôt est obtenu majoritairement au voisinage du point d'impact (200 $\mathrm{m}$ dans les graphiques).

Le caractère très asymétrique des dépôts finaux (paramètre essentiel pour

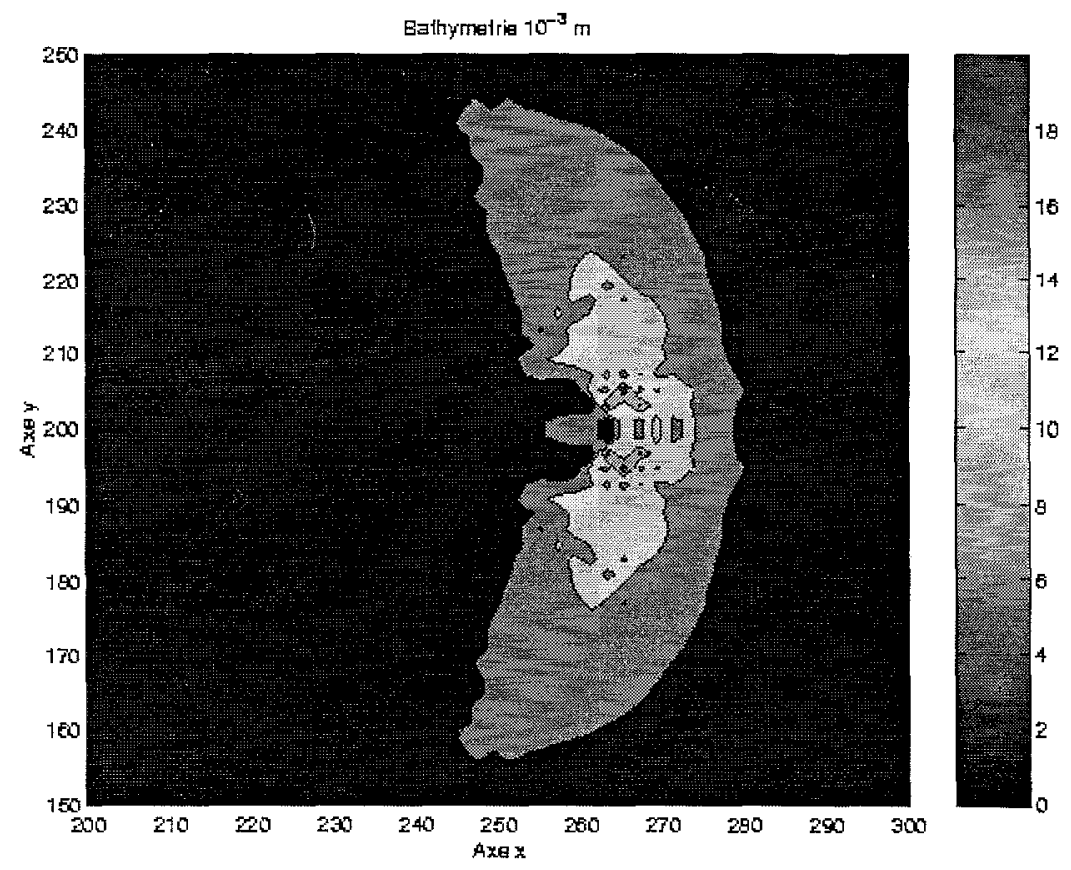

Figure 6 - Variation bathymétrique après 20 min de simulation. 
l'évolution à long terme) en présence de courant est illustré par la figure 6 , où sont montrés les dépôts après 20 min de simulation ainsi que l'évolution temporelle du pourcentage de matière turbide restant dans le courant de turbidité.

Ces résultats montrent l'effet notoire de la présence d'un courant ambiant sur la déposition de la masse turbide d'un rejet de dragage. Toutefois, il faut remarquer que ce courant est cependant relativement faible $\left(10 \mathrm{~cm} . \mathrm{s}^{-1}\right)$ car il rend possible le dépôt de la masse turbide.

Les résultats obtenus pour un fond non horizontal (pente faible et constante) ont montré une asymétrie mais qui semble moins importante par rapport à celle induite par un courant ambiant.

\section{Conclusions et perspectives}

L'approche de modélisation développée semble prometteuse pour la simulation du devenir à court terme d'un rejet de dragage par clapage. Le couplage avec des modèles simulants les cinétiques propres aux contaminants associés permettrait dans un futur proche d'estimer les flux de contaminants sortant de la zone de rejet. En outre, les résultats de cette approche de modélisation permettent d'introduire des conditions initiales dans un modèle classique d'advection-dispertion et de transport sédimentaire dans l'objectif d'estimer l'étendue locale ou régionale des zones impactées.

La recherche présentée est toujours en cours, mais on observe avec les premiers résultats que lors d'une application opérationnelle, toujours contrainte par un besoin de simplification, qu'il est nécessaire de tenir compte de la présence d'un courant ambiant, ce qui était relativement mal pris en compte dans les modèles opérationnels disponibles (e.g. les modèles de 1'USACE). Par contre la présence d'une pente et d'une irrégularité du fond et son éventuelle simplification ne semble pas avoir d'effets très importants.

La chaîne de modélisation présentée (modèle de convection, puis d'étalement) suivie de l'utilisation d'un modèle d'advection-dispersion classique permettrait ainsi d'aborder les applications opérationnelles dans le court terme. Son utilisation reste cependant limitée aux situations où l'hydrodynamisme ambiant permet quelques simplifications. Aussi, il est nécessaire de concevoir des expériences in situ ou des protocoles de suivi de rejets réels de manière à valider ce type de modélisation.

D'autres modèles plus sophistiqués, avec une physique plus étendue et capables de simuler toutes les phases d'un rejet, sont déjà proposés ou en cours de développement: il s'agit de modèles non hydrostatiques tri-dimentionnels qui demeurent cependant trop coûteux pour une application opérationnelle. Ces modèles constituent et constitueront toutefois des outils précieux pour une calibration et une validation d'approches plus simples comme celle que nous proposons.

\section{Bibliographie}

Abdelrhman M.A. et E.H. Dettmann, 1993. Dredged material transport at deep-sea disposal sites. Coastal Engineering Considerations in Coastal Zones 


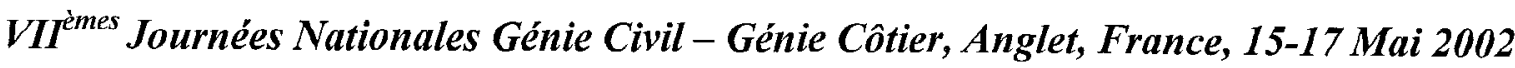

Management. Proccedings, $8^{\text {th }}$ Symposium on Coastal and Ocean Management, New Orleans, Louisiana, July 19-23, 216-230.

Alzieu C., P. Michel, J.-F. Chiffoleau, B. Boutier et A. Abarnou, 1999. Contamination chimique des sédiments. Dans Dragages et environnement marinEtat des connaissances, coordinateur Claude Alzieu, 70-110.

Boutin R., 2000. Dragage et Rejets en Mer, les produites de type vase. Presses de 1'Ecole Nationale des Ponts et Chaussées, ISBN 2-85978-336-9, 307 pp.

Bradford S.F., N.D. Katapodes et G. Parker, 1997. Characteristic analysis of turbid underflows. Journ. of Hydraulic Eng., may 1997, 420-431.

Brandsma M.G. et D.J. Divoky, 1976. Development of models for prediction of short-term fate of dredged material discharged in the estuarine environment. Contract report D-76-5, US Army Engineer Waterways Experiment Station, Vicksburg, MS. Prepared by Tetra Tech. Inc., Pasadena, CA.

Burel D. et A. Garapon, 2002. 3D numerical modeling of dredged material descent. International Conference in Coastal Engineering, ICCE'2002 (résumé accepté).

Gotoh H. et J. Fredsoe, 2000. Lagrangian two phase flow model of the settling behavior of fine sediment dumped into water. Proc. Int. Coastal Engineering Conference. July 2000 Sydney, Australia, 4, 3907-3919.

Johnson B.H., Scheffner N.W., Teeter A.M., Hands E.B., Moritz H.R., 1995. Anamysis of dredged material placed in open water. Dredging' $94.2^{\text {nd }}$ Int. Conf. On dredging and Dredged Material Placement, Buena vista, FL, 1, 18-27.

Koh R.C.Y. et Y.C. Chang, 1973. Mathematical model for barged ocean disposal of waste. Technical series EPA 660/2-73-029, U.S. Environmental Protection Agency, Washington, DC.

L'Yavanc J., C. Alzieu et J.-L. Mauvais, 1999. Les dragages sur le littoral français. Dans Dragages et environnement marin - Etat des connaissances, coordinateur Claude Alzieu, Ed. Ifremer ISBN 2-84433-014-2, 27-38.

McAnally W.H.Jr et S. Adamec, 1987. Designing open water disposal for dredged muddy sediments. Con. Shelf Res., 7, 11/12, 1445-1455.

Parker G., Y. Fukushima et H.M. Pantin, 1986. Self-accelerating turbidity currents over an erodible bed. J. Hyd. Res., 25(1), 123-147.

Silva Jacinto R., P. Le Hir et P. Bassoullet, 1999. Modélisation mathématique des rejets de dragage. Dans Dragages et environnement marin Etat des connaissances, coordinateur Claude Alzieu, 152-168.

Teeter A.M., 1984. Vertical transport in fine-grained suspension and newly-deposited sediment. Lecture Notes on Coastal and Estuarine Cohesive Sediment Dynamics. Mehta A.J. (ed.), Springer-Verlag, NY.

Truitt C.L., 1988. Dredge material behaviour during open water disposal. J. Coast. Res., 4, 3, 389-397. 